OPEN ACCESS

Edited by:

Jose Eduardo Villarreal Barajas, Royal Devon and Exeter Hospital, United Kingdom

Reviewed by: Kundan Thind,

Alberta Health Services, Canada Weiwei Zong,

Henry Ford Health System, United States

${ }^{*}$ Correspondence:

Hui Wu

terrywuhui@sina.com Guangming Niu Cjr.niuguangming@vip.163.com

Specialty section: This article was submitted to

Radiation Oncology,

a section of the journal

Frontiers in Oncology

Received: 27 August 2021 Accepted: 04 October 2021 Published: 21 October 2021

Citation:

YuXY, Ren J, Jia Y, Wu H, Niu G, Liu A, Gao Y, Hao F and Xie L (2021) Multiparameter MRI Radiomics Model Predicts Preoperative Peritoneal Carcinomatosis in Ovarian Cancer.

Front. Oncol. 11:765652. doi: 10.3389/fonc.2021.765652

\section{Multiparameter MRI Radiomics Model Predicts Preoperative Peritoneal Carcinomatosis in Ovarian Cancer}

\author{
Xiao Yu Yu ${ }^{1}$, Jialiang Ren ${ }^{2}$, Yushan $\mathrm{Jia}^{3}$, Hui $\mathrm{Wu}^{3 *}$, Guangming Niu ${ }^{3 *}$, Aishi Liu ${ }^{3}$, \\ Yang $\mathrm{Gao}^{3}$, Fene $\mathrm{Hao}^{3}$ and Lizhi Xie ${ }^{3}$ \\ ${ }^{1}$ Affiliated Hospital, Inner Mongolia Medical University, Hohhot, China, ${ }^{2}$ Department of Pharmaceuticals Diagnosis, GE \\ Healthcare (China), Shanghai, China, ${ }^{3}$ Department of Radiology, Inner Mongolia International Hospital, Hohhot, China
}

Objectives: To evaluate the predictive value of radiomics features based on multiparameter magnetic resonance imaging (MP-MRI) for peritoneal carcinomatosis $(\mathrm{PC})$ in patients with ovarian cancer (OC).

Methods: A total of 86 patients with epithelial OC were included in this retrospective study. All patients underwent FS-T2WI, DWI, and DCE-MRI scans, followed by total hysterectomy plus omentectomy. Quantitative imaging features were extracted from preoperative FS-T2WI, DWI, and DCE-MRI images, and feature screening was performed using a minimum redundancy maximum correlation (mRMR) and least absolute shrinkage selection operator (LASSO) methods. Four radiomics models were constructed based on three MRI sequences. Then, combined with radiomics characteristics and clinicopathological risk factors, a multi-factor Logistic regression method was used to construct a radiomics nomogram, and the performance of the radiomics nomogram was evaluated by receiver operating characteristic curve (ROC) curve, calibration curve, and decision curve analysis.

Results: The radiomics model from the MP-MRI combined sequence showed a higher area under the curve (AUC) than the model from FS-T2WI, DWI, and DCE-MRI alone (0.846 vs. 0.762, 0.830, 0.807, respectively). The radiomics nomogram (AUC=0.902) constructed by combining radiomics characteristics and clinicopathological risk factors showed a better diagnostic effect than the clinical model $(A \cup C=0.858)$ and the radiomics model (AUC=0.846). The decision curve analysis shows that the radiomics nomogram has good clinical application value, and the calibration curve also proves that it has good stability.

Conclusion: Radiomics nomogram based on MP-MRI combined sequence showed good predictive accuracy for PC in patients with OC. This tool can be used to identify peritoneal carcinomatosis in OC patients before surgery.

Keywords: ovarian cancer, peritoneal carcinomatosis, radiomics, magnetic resonance imaging, predictions 


\section{INTRODUCTION}

Ovarian cancer (OC) is the fifth most common cancer in women and the most common gynecological tumor. Epithelial ovarian cancer (EOC) is the most common OC subtype accounting for $90 \%$ of all OC. It is characterized by extensive and rapid intra-abdominal carcinomatosis and has a poor prognosis and high mortality. The 5-year survival rate of EOC is only $30 \%(1-4)$. If the patient can detect PC at an early stage, it will be able to buy sufficient treatment time for the patient and effectively control the patient's condition from further deterioration. Preoperative detection of peritoneal carcinomatosis (PC) is essential to avoid unnecessary resection and choose the best treatment method for patients with EOC.

National Comprehensive Cancer Network guidelines recommend that all peritoneal surfaces suspected of carcinomatosis should be selectively removed. Many ovarian cancers do not have ascites when they have peritoneal carcinomatosis. Because there are many peritoneal folds, smaller peritoneal metastatic nodules can be easily misdiagnosed, which affects the treatment and prognosis of patients. The diagnosis of peritoneal implants mainly relies on open exploratory surgery and laparoscopy; nonetheless, the existing results are hardly consistent. In addition, laparoscopic surgery, which is invasive and expensive, carries certain risks, such as intraoperative tumor capsule rupture, incision carcinomatosis $(5,6)$. Therefore, there is an urgent need for an accurate non-invasive technique to assess the PC's condition.

As an alternative method, computed tomography (CT) is usually used for preoperative examination. However, CT has limited sensitivity and may easily overlook carcinomatosis below $1 \mathrm{~cm}$ (7). Radiomics, an emerging and promising research field based on quantitative imaging technology, can provide decision support for oncology by extracting highthroughput quantitative radiological features from medical images (8). This low-cost and non-invasive technique has been successfully used for tumor diagnosis, staging, treatment monitoring, and treatment plan formulation (9-12). This method has been successfully applied to preoperatively predict peritoneal carcinomatosis of gastric cancer $(13,14)$ and may be potentially used for ovarian cancer. So far, no personalized prediction model has been developed for peritoneal carcinomatosis of ovarian cancer. This study evaluated the value of multi-parameter MRI radiomics in predicting preoperative peritoneal carcinomatosis in patients with ovarian cancer. We established a combined clinical-radiomics model to help improve decision-making and guide individualized treatment.

\footnotetext{
Abbreviations: OC, ovarian cancer; EOC, epithelial ovarian cancer; PC, peritoneal carcinomatosis; AJCC, American Joint Committee on Cancer; MP-MRI, multiparameter magnetic resonance imaging; ROI, region of interest; ICC, inter-group correlation coefficient; AUC, area under the curve; DCA, decision curve analysis.
}

\section{MATERIALS AND METHODS}

\section{Patient Information}

This retrospective study was approved by the ethical review committee of our hospital, and informed consent was obtained from patients.

From June 2015 to May 2020, 350 consecutive EOC patients were retrieved retrospectively in our hospital's image archiving and communication system (PACS, GE). The inclusion/ exclusion criteria and patient recruitment process are shown in Figure 1. Inclusion criteria: 1) Histopathologically confirmed epithelial ovarian cancer; 2) Receive MRI examination one week before surgery. Exclusion criteria were: 1) past treatment history of ovarian cancer $(n=69)$; 2) the histopathology was nonepithelial OC $(n=39) ; 3)$ no dynamic enhanced MRI of the pelvis before treatment $(\mathrm{n}=34)$; 4) presence of clear PC signs on pelvic MRI $(n=45)$; 5) the clinical data of CA125 were incomplete $(\mathrm{n}=31) ; 6)$ there are other distant carcinomatosis $(\mathrm{n}=46)$. Finally, 86 patients (age 33-82, median age 54) were enrolled in the study.

All patients underwent full hysterectomy with double appendages and increased omentectomy. The existence of PC was unanimously determined by pathologists and gynecologists according to AJCC (American Joint Committee on Cancer) guidelines. Finally, 39 out of 47 patients were detected with peritoneal carcinomatosis. The clinicopathological characteristics, including age, preoperative CA125 level, abdominal symptoms, menopausal history, genetic history, and type, were obtained from patients' medical records.

Two radiologists with 3 and 15 years of experience in female pelvic MRI imaging, who were blind to the pathological results but knew whether the patient was diagnosed with EOC, reviewed the MRI images and recorded the following: (1) unilateral or bilateral ovarian tumors; (2) tumor size (the volume of the largest layer); (3) T2 signal (low and high signals were divided by the signal strength near the myometrium); (4) enhancement degree. The radiological characteristics were selected according to the criteria of Guo HL et al. (15). Bilateral lesions were determined using the same pathological type according to the pathological surgical results; the largest tumor is finally selected for analysis. The ADC value was obtained according to the method of Thomassin et al. (16). The clinical and tumor characteristics of the patients are summarized in Tables $\mathbf{1}, \mathbf{2}$.

\section{Imaging Acquisition and Preprocessing}

All MRI examinations were performed on a 3.0T system (Discovery MR750, GE Healthcare), using an 8-channel phased body coil. Before scanning, the patient with moderately filled bladder was placed in a supine position. Patients were also asked to fast 4-6 hours before the examination, and intestinal preparation (lactulose and magnesium sulfate) was used to reduce bowel movements. Scans were then performed along the pubic bone to the iliac spine. MR scanning parameters on the 3.0-T scanner DCE imaging of the pelvis was performed after administration of $0.1 \mathrm{mmol} / \mathrm{kg}$ of body weight of gadolinium chelate (Gadovist; Bayer). Images were acquired at postcontrast 


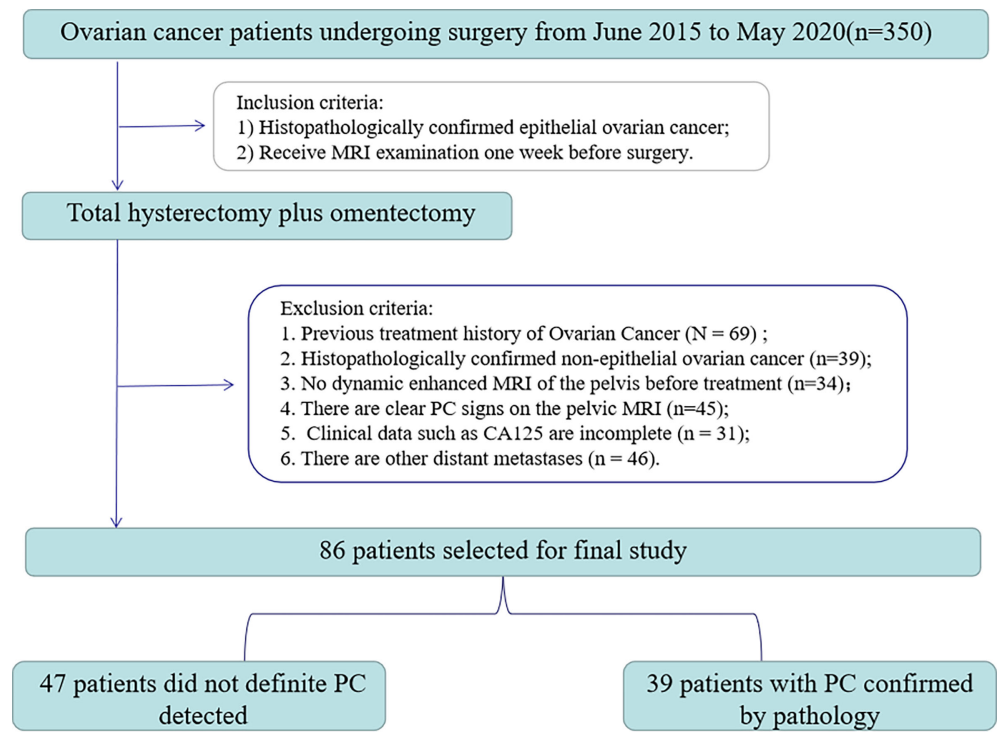

FIGURE 1 | Flowchart of patient selection.

TABLE 1 | Single-factor analysis of clinicopathological characteristics of 86 EOC patients.

\begin{tabular}{|c|c|c|c|}
\hline Features & Without PC & With PC & $p$ value \\
\hline Number of patients & 47 & 39 & \\
\hline Age (mean $\pm \mathrm{SD}$, years) & $51.7 \pm 8.8$ & $56.8 \pm 10.6$ & 0.017 \\
\hline CA125 (median $\pm \mathrm{IQR}, \mu / \mathrm{ml})$ & $213.1(75.0-397.4)$ & 1237. (608.7-2247.9) & $<0.001$ \\
\hline Genetic history (\%) & & & 0.950 \\
\hline Yes & 7 (14.9\%) & 6 (15.4\%) & \\
\hline No & $40(85.1 \%)$ & $33(84.6 \%)$ & \\
\hline Menopause(\%) & & & 0.381 \\
\hline Yes & $27(57.4 \%)$ & $26(66.7 \%)$ & \\
\hline No & $20(42.6 \%)$ & $13(33.3 \%)$ & \\
\hline Abdominal symptoms (\%) & & & 0.988 \\
\hline Yes & $29(61.7 \%)$ & $24(61.5 \%)$ & \\
\hline No & 18 (38.3\%) & 15 (38.5\%) & \\
\hline Type (\%) & & & 0.087 \\
\hline Type I & $19(40.4 \%)$ & $9(23.1 \%)$ & \\
\hline Type II & $28(59.6 \%)$ & $30(76.9 \%)$ & \\
\hline
\end{tabular}

$S D$, standard deviation; IOR, interquartile range; $A D C$, apparent diffusion coefficient; $P C$, peritoneal metastasis.

TABLE 2 | Single-factor analysis of MR imaging characteristics of 86 EOC patients.

\begin{tabular}{|c|c|c|c|}
\hline Features & Without PC & With PC & $p$ value \\
\hline Size (median \pm IQR, mm3) & 884.7 (239.9-1123.8) & 596.4 (120.0- 844.2) & 0.093 \\
\hline $\mathrm{ADC}$ (Average $\pm \mathrm{SD}, \mathrm{mm} 2 / \mathrm{s}$ ) & $0.001 \pm 0.001$ & $0.001 \pm 0.000$ & 0.088 \\
\hline Location (\%) & & & 0.123 \\
\hline Unilateral & $34(72.3 \%)$ & $22(56.4 \%)$ & \\
\hline Bilateral & $13(27.7 \%)$ & 17 (43.6\%) & \\
\hline T2 homogeneity (\%) & & & 0.203 \\
\hline Low & 14 (29.8\%) & $7(17.9 \%)$ & \\
\hline High & $33(70.2 \%)$ & $32(82.1 \%)$ & \\
\hline T1 enhancement (\%) & & & 0.057 \\
\hline Mild & $20(42.6 \%)$ & $9(23.1 \%)$ & \\
\hline Obvious & $27(57.4 \%)$ & $30(76.9 \%)$ & \\
\hline
\end{tabular}

$S D$, standard deviation; IOR, interquartile range; $A D C$, apparent diffusion coefficient; $P C$, peritoneal metastasis. 
enhancement 120 seconds in the axial plane. This protocol obtained axial FS-T2WI, DWI, and DCE-MRI images. Detailed information about the acquisition parameters is shown in Table S1.

Before image segmentation, preprocessing was required. First, according to the research of Qian et al. (17), the fs-T2WI and DWI sequence images of each patient were selected for registration to the DCE-MRI (late arterial stage only) image. Then, the planar resolution of each mode was uniformly resampled to $1 \times 1 \times 1 \mathrm{~mm}$. Finally, the method of Cohen (18) was applied to normalize the image contrast of each mode to correct the factors that may affect the intensity unevenness. All the processing was performed on the 3D Slicer (version 4.10.2, funded by the National Institutes of Health) software.

\section{MRI Radiomics Feature Extraction and Selection}

Ovarian cancer lesions were performed by two radiologists with 3 years (A) and 15 years (B) experience in abdominal imaging respectively on each layer of DWI $(b=1000 \mathrm{~s} / \mathrm{mm} 2)$ to perform 3D manual manipulation of the primary tumor along the edge of the lesion segmentation. The region of interest (ROI) covered the entire tumor. The FS-T2WI and DCE-MRI were compared to avoid the cystic, necrotic, or hemorrhage area of the tumor (see Appendix 1).

PyRadiomics was used (19) to extract the radiologic signatures. Wavelet (8 filtering parameters) and Laplace of Gaussian (LoG, 2 filtering parameters) transformations were applied on the original image, respectively. Then, 1037 features were extracted from 11 different image types, including (1) gray histogram features; (2) morphological features; (3) gray level cooccurrence matrix (GLCM) features; (4) gray level run length matrix (GLRLM) functions; (5) grayscale area matrix (GLSZM) features. After that, all eigenvalues were normalized using
Z-Score transformation. In order to ensure the reproducibility of the model results and reduce the over-fitting or selection bias in the radiomics model, the intra- and inter-group correlation coefficient (ICC) was used to evaluate the characteristics of retention stability and high repeatability, and the ICC threshold was set to 0.75 . Then, the minimum redundancy maximum correlation (mRMR) was used to sort the remaining features, and each sequence retained the best top 20 features (2022). Next, the least absolute shrinkage selection operator (LASSO) (23) method was used to screen the radiological features used to evaluate PC status. Finally, multi-factor stepwise logistic regression was used, and the Akaike Information Criterion (AIC) was used as the stopping condition to determine the best combination of radiological characteristics and clinical data (23). The workflow is shown in Figure 2.

\section{Establishment of Radiomics and Clinical Models}

The radiomic characteristics screened by the above method were incorporated into the multivariate Logistic regression analysis to establish a radiomics model. All the above steps were performed on the radiomics model extracted from FS-T2WI, DWI, and DCE-MRI separately and the combined model. In addition, for comparison, a Logistic regression analysis model containing clinical data was also established. Finally, the radiomics features were combined with clinical data to construct a hybrid model. In order to provide visualization and a personalized tool for predicting the probability of ovarian cancer peritoneal carcinomatosis, we have drawn a nomogram. The calibration curve was used to evaluate the calibration of the nomogram, and the Hosmer-Lemeshow test was performed. Decision curve analysis was used to calculate the net income of different models under different threshold probabilities.

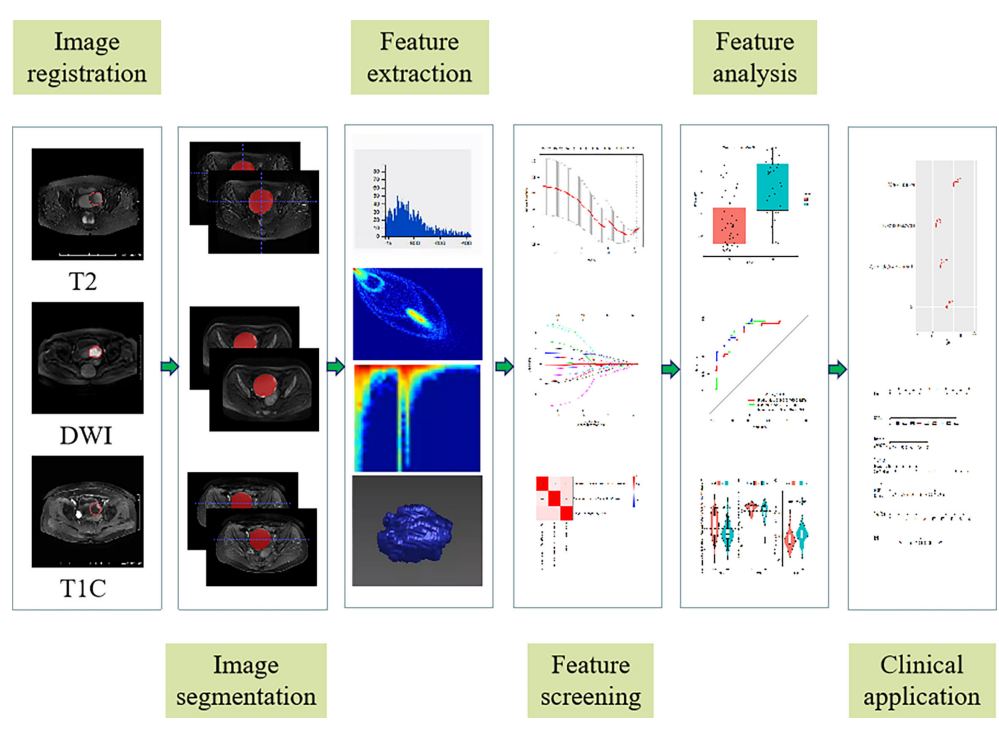

FIGURE 2 | Radiomics signature workflow. 


\section{Statistical Analysis}

All statistical analyses were performed using R (Version 3.6.3, Statistical Computing Basis). Independent sample t-test or Mann-Whitney $U$ test were used to examine the differences in measurement data uses, and the chi-square test or Fisher's exact test to evaluate the count data differences. The receiver operating characteristic curve (ROC) of the model was drawn, and the area under the curve (AUC) and 95\% confidence interval (CI) were calculated to quantify the discriminant ability of the model. Delong test was used to compare the AUC among different models. The diagnostic sensitivity, specificity, accuracy, positive likelihood ratio, and negative likelihood ratio were also examined. Calibration curves were used to evaluate the predictive performance of each model. Decision curve analysis (DCA) was used to evaluate the net benefits of each model under different threshold probabilities and to evaluate the clinical applicability of each model. A two-sided p-value less than 0.05 was considered to be statistically significant.

\section{RESULTS}

\section{Clinical and Tumor Characteristics of Patients}

The clinicopathological and radiological characteristics of the patients are shown in Tables $\mathbf{1}$ and $\mathbf{2}$. There were significant differences in age and preoperative CA125 levels between ovarian cancer (OC) with PC and OC without PC $(\mathrm{p}=<0.001-0.017)$. However, no differences were found for abdominal symptoms, menopausal history, genetic history, type, lesion location, tumor size, T2 signal, and ADC between the two groups.

\section{Evaluation of Radiomics}

According to the standard of ICC $>0.75$, FS-T2WI, DWI, and DCE-MRI sequences retained 508, 557, and 508 radiomic features, respectively. Minimum redundancy maximum correlation and Lasso regression were then performed on the selected omics features to adjust penalty parameters through 10 cross-validation and select non-zero coefficient features related to PC status. Finally, 2 FS-T2WI features, 1 DWI feature, and three DCE-MRI features were used to build a model (Appendix Figure 2). Consequently, after removing features by multivariate Logistic regression, 3 features were retained, and a combined multi-sequence model was established (Appendix Figure 3).

The combined model from multiple sequences showed better distinguishing ability than the single model (when using a single sequence). The ROC curves of the four models are shown in Figure 3A. The AUC values of Fs-T2WI, DWI, CE-T1WI, and the combined model were $0.762(0.662-0.861), 0.830(0.745-$ $0.914), 0.807$ (0.717-0.898), and 0.846(0.765-0.927), respectively. The performance of the models is shown in Table S2. The DCA curves and calibration curves of the four models are shown in Appendix Figure 4.

\section{Model Comparison and Nomogram Performance}

Multivariate analysis of clinical data and radiomic characteristics showed that preoperative CA125 level, DWI_HLH_glszm_Size ZoneNonUniformityNormalized, T1C_glszm_LowGrayLevel ZoneEmphasis, T1C_LHL_ngtdm_Contrast were significant predictors (Figure 4). As a result, they were fused into a nomogram (Figure 5). The AUC of the radiology nomogram was higher than that of the clinical model and the radiomic model (0.902, 95\%CI: 0.846-0.858), indicating that the radiology nomogram can effectively distinguish the presence or absence of peritoneal carcinomatosis. The predictive performance of the clinical model was not significantly different from that of the omics model ( $\mathrm{AUC}=0.858$ vs. $\mathrm{AUC}=0.846$ ). Figure 3B summarizes the diagnostic performance and ROC analysis results of these three models.

The calibration curve of the nomogram showed a good agreement between the predicted value and the observed value.
A

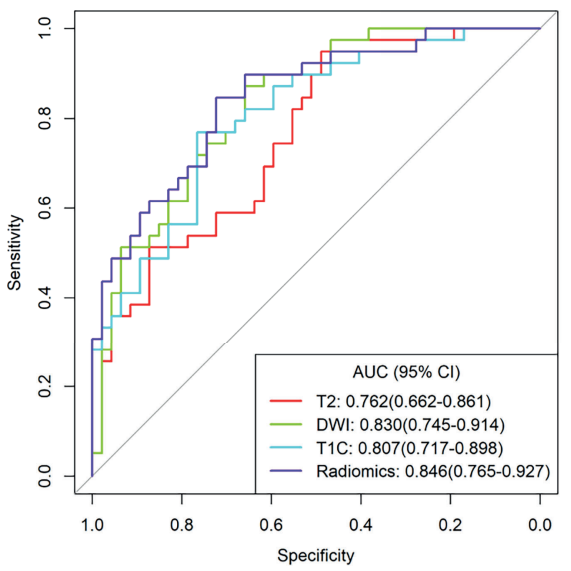

B

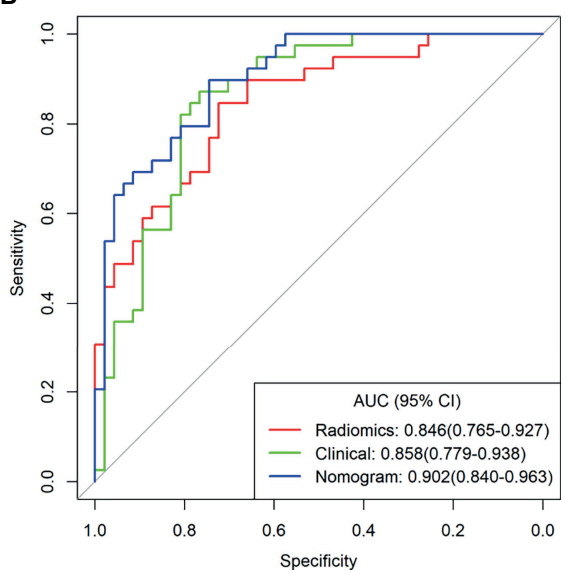

FIGURE 3 | (A) T2WI radiation model, DWI radiation model, T1C Radiation Model, and combined radiation model. (B) Clinical Model, combined radiological model, and Nomogram Receiver Operating Characteristic curve. 


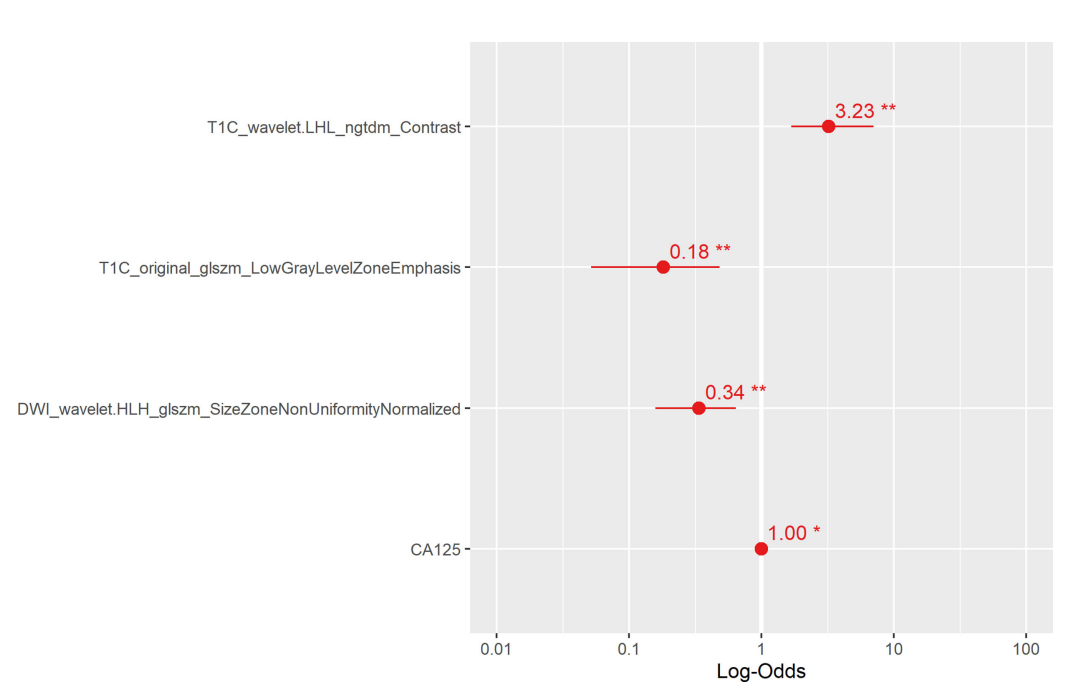

FIGURE 4 | The main radiological features extracted in this study and the results of multiple logistic regression of preoperative CA125. The horizontal line is the $95 \%$ confidence interval of the study, and the small dot in the center of the horizontal line is the point of the OR value.

The Hosmer-Lemeshow test was not significant ( $\mathrm{p}>0.05)$, indicating a good degree of fit (Figure 6A). Decision curves were used to compare the benefits of nomograms, radiomics models, and clinical models, and we found that when the threshold probability of DCA curves was $37 \%-85 \%$, nomograms had better predictive performance than clinical models and omics models (Figure 6B).

\section{DISCUSSION}

In this study, a nomogram radiomics model for preoperative prediction of peritoneal carcinomatosis of EOC was proposed based on clinical data and radiomics features reflecting primary tumors' characteristics. Our research shows that the multisequence combination model is better than the single-sequence

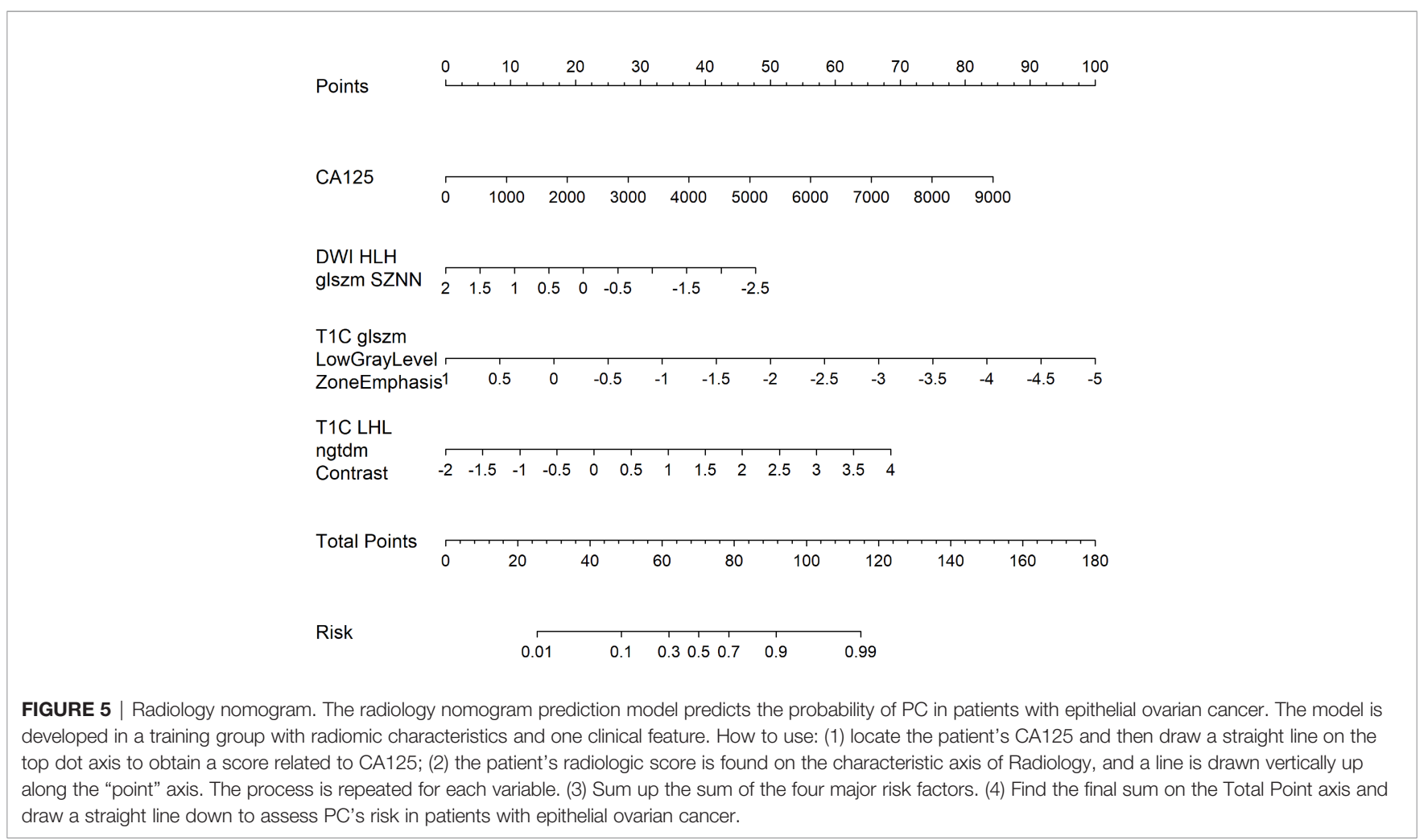


A

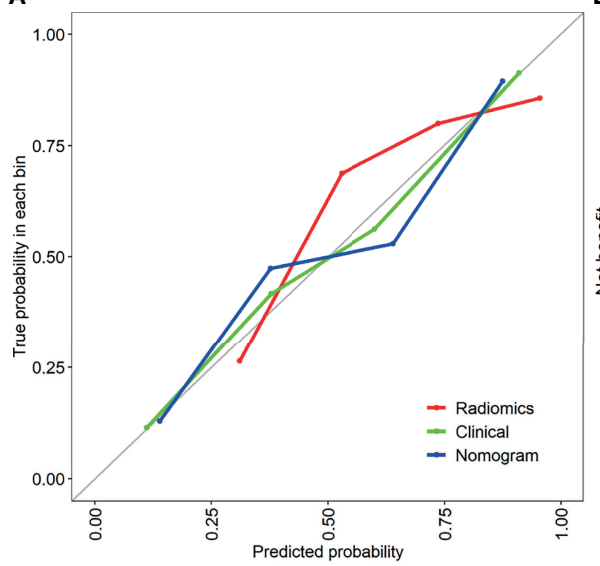

B

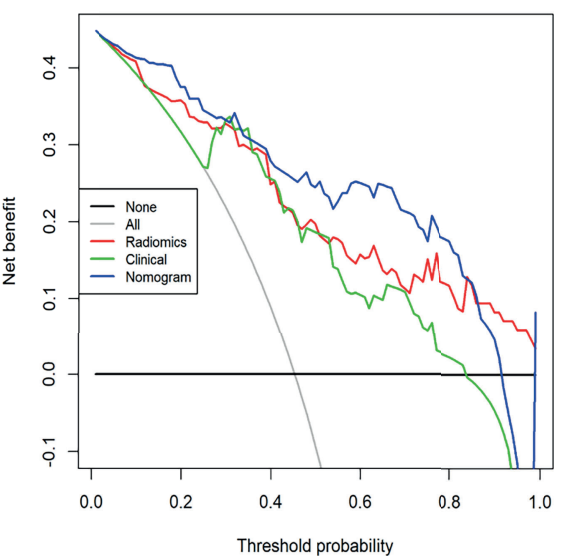

FIGURE 6 | (A) The calibration curve of the clinical model, combined radiology model, and nomogram. It is a curve with the model predicted PC probability as the $\mathrm{X}$-axis and the actual PC probability as the $\mathrm{Y}$-axis. The degree of coincidence between the calibration curve depicted and the 45 -degree straight line reflects the predictive performance of each model. (B) Decision curve analysis of the clinical model, combined radiomics model, and mixed model. The $Y$-axis represents net income. The blue line represents the radiographic nomogram. Red lines represent radiomics models. The green line represents the model that contains only clinical features. The gray line represents the assumption that all patients have LN metastasis. The thin black line indicates the hypothesis that no patients have PC metastasis.

model. The constructed nomogram provides an easy-to-use, non-invasive and individualized tool for PC diagnosis and provides decision support for clinicians.

Some studies have explored the value of MRI in the evaluation of PC in epithelial ovarian cancer. At present, all published studies, including recent studies, have been conducted with comparable or fewer patient sample sizes, mainly focusing on routine imaging $(15,24,25)$. Our study extracted more than 3000 features from MP-MRI images and evaluated the MR imaging features of patients with epithelial ovarian cancer in FS-T2WI, DWI, DCE-MRI, and the combination of the three. When FS-T2WI, DWI, and DCE-MRI were combined, the diagnostic efficiency of the presence or absence of peritoneal carcinomatosis was the highest, which is consistent with previous reports in the literature $(26,27)$. The DWI of our study is very close to the AUC of radiomics. This is because in the absence of ascites, some small lesions are usually better seen on the DWI image than on the standard T1 and T2 weighted images. To the best of our knowledge, this is the first study that combines anatomical, diffusion, and perfusion MRI and uses radiomics analysis based on the primary tumor to predict PC. Therefore, the MP-MRI combined model reveals more detailed tumor information and can more accurately predict ovarian cancer's PC status.

In our study, preoperative CA125 level was considered to be an independent predictor of PC carcinomatosis. Age has a certain potential for predicting PC, but it is not as effective as CA125. Therefore, a clinical model based on CA125 was established. Our data showed that the risk of PC with high levels of CA125 was significantly higher, which is consistent with the results reported in the previous literature $(28,29)$. At the same time, in order to facilitate clinical use, we have developed a nomogram containing preoperative CA125 levels and radiomic characteristics, with an
AUC value of 0.902 , for predicting PC. The DCA curve showed a satisfactory net income. The calibration curve showed that the stability was slightly inferior to the clinical model. This is because the clinical model was established based on a single variable, and CA125 was a continuous variable with a small fluctuation range. The nomogram integrates clinical data and radiomics characteristics and contains multi-dimensional quantitative and detailed information, so the results obtained are more objective and accurate.

Peritoneal carcinomatosis are common in the stomach, gallbladder, pancreas, lungs, intestines, uterus, and ovaries (3032). Most of the patients with early-stage ovarian cancer PC have no specific symptoms; these patients are usually diagnosed when in an advanced stage. It is one of the main causes of ovarian cancer morbidity and mortality (32). So far, many studies have evaluated the PC status of patients with ovarian cancer $(15,24$, 25). CT is a common tool used to detect PC, but its missed diagnosis rate is high. Although ${ }^{18} \mathrm{~F}-\mathrm{FDG}$ PET/CT has been shown to achieve good results in evaluating PC status, it is not widely used because of the high cost. MRI has a high resolution for soft tissues and can clearly show anatomical relationships.

Studies have recently shown that radiomics can predict peritoneal carcinomatosis in cancer patients $(13,14)$. Dong et al. (13) developed a personalized nomogram to identify the occult peritoneal carcinomatosis of advanced gastric cancer, achieving good results, which is similar to our findings, with few differences: first, we used MP-MRI to extract features, which can reflect tumor information more comprehensively and in more detail; second, we established a single sequence model and a combined model. Through comparison, we found that the combined model had better performance in predicting PC. In addition, the nomogram we established also showed good clinical practicability and ability to provide a diagnostic basis for predicting the PC status of ovarian cancer before clinical surgery. 
This study has a few limitations. First, it is a retrospective study with small sample size. In the future, more patients are needed to provide more reliable evidence for clinical applications. Secondly, the research model's establishment and verification is a single-center, and further data from multiple centers are needed for external validation. In addition, we only performed radiomics analysis on late-arterial phase of contrastenhanced MRI. Perhaps venous MRI images may provide more useful radiomics information, which needs to be discussed further. Finally, only primary tumors were selected in our study, and the radiohistological features of the peritoneum were not routinely used. MRI texture analysis of peritoneum needs to be further studied to explore its value.

In summary, we established nomograms based on preoperative CA125 and radiographic characteristics from primary tumors, which can be used to predict peritoneal carcinomatosis in EOC patients preoperatively. This effective and easy-to-use new approach provides a non-invasive and reliable tool for EOC patients to develop individualized treatment plans.

\section{DATA AVAILABILITY STATEMENT}

The original contributions presented in the study are included in the article/Supplementary Material. Further inquiries can be directed to the corresponding authors.

\section{ETHICS STATEMENT}

The studies involving human participants were reviewed and approved by Biomedical Medical Research Ethics Committee of

\section{REFERENCES}

1. Siegel RL, Miller KD, Jemal A. Cancer Statistics, 2018. CA Cancer J Clin (2018) 68(1):7-30. doi: 10.3322/caac.21387

2. Scalici JM, Atkins KA, Petroni G, Duska RL, Atkins AK, Saks JE, et al. Mesothelium Expression of Vascular Cell Adhesion Molecule-1 (VCAM-1) Is Associated With an Unfavorable Prognosis in Epithelial Ovarian Cancer (EOC). Cancer (2017) 23(6):977-84. doi: 10.1002/ cncr.30415

3. Ahmed N, Stenvers Kaye L. Getting to Know Ovarian Cancer Ascites: Opportunities for Targeted Therapy-Based Translational Research. Front Oncol (2013) 3(3):256. doi: 10.3389/fonc.2013.00256

4. Cannistra, Stephen A. Cancer of the Ovary. N Engl J Med (2004) 351 (24):1550-9. doi: 10.1056/NEJMra041842

5. Smorgick N, Barel O, Halperin R, Schneider D, Pansky M. Laparoscopic Removal of Adnexal Cysts: Is it Possible to Decrease Inadvertent Intraoperative Rupture Rate? Am J Obstet Gynecol (2009) 200(3):237.e1-3. doi: 10.1016/j.ajog.2008.10.030

6. Zivanovic O, Sonoda Y, Diaz John P, Levine DA, Brown CL, Chi DS, et al. The Rate of Port-Site Metastases After 2251 Laparoscopic Procedures in Women With Underlying Malignant Disease. Gynecol Oncol (2008) 111(3):431-7. doi: 10.1016/j.ygyno.2008.08.024

7. Suidan Rudy S, Ramirez Pedro T, Sarasohn Debra M, Sarasohn, Jerrold B, Teitcher, et al. A Multicenter Prospective Trial Evaluating the Ability of Preoperative Computed Tomography Scan and Serum CA-125 to Predict Suboptimal Cytoreduction at Primary Debulking Surgery for Advanced
Inner Mongolia Medical University. The patients/participants provided their written informed consent to participate in this study.

\section{AUTHOR CONTRIBUTIONS}

$\mathrm{XY}$ and JR substantial contributions to the conception or design of the work; or the acquisition, analysis or interpretation of data for the work. HW and GN drafting the work or revising it critically for important intellectual content. YJ, FH, and LX provide approval for publication of the content. YG and AL agree to be accountable for all aspects of the work in ensuring that questions related to the accuracy or integrity of any part of the work are appropriately investigated and resolved. All authors contributed to the article and approved the submitted version.

\section{FUNDING}

This article was funded by the Inner Mongolia Autonomous Region Fund of Natural Science (2021MS08026).

\section{SUPPLEMENTARY MATERIAL}

The Supplementary Material for this article can be found online at: https://www.frontiersin.org/articles/10.3389/fonc.2021.765652/ full\#supplementary-material

Ovarian, Fallopian Tube, and Peritoneal Cancer. Gynecol Oncol (2014) 134 (3):455-61. doi: 10.1016/j.ygyno.2014.07.002

8. Gillies Robert J, Kinahan Paul E, Hricak H. Radiomics: Images Are More Than Pictures, They Are Data. Radiology (2016) 278(2):563-77. doi: 10.1148/ radiol.2015151169

9. Drukker K, Giger ML, Joe BN, Kerlikowske K, Shepherd. Combined Benefit of Quantitative Three-Compartment Breast Image Analysis and Mammography Radiomics in the Classification of Breast Masses in a Clinical Data Set. Radiology (2018) 290(3):180608. doi: 10.1148/ radiol.2018180608

10. Park Hyo J, Lee Seung S, Park B, Yun J, Sung YS, Shim WH, et al. Radiomics Analysis of Gadoxetic Acid-Enhanced MRI for Staging Liver Fibrosis. Radiology (2019) 292(1):269. doi: 10.1148/radiol.2019194012

11. Mattonen SA, Davidzon GA, Benson J, Leung ANC, Nair VS, Horng G, et al Bone Marrow and Tumor Radiomics at 18F-FDG PET/CT: Impact on Outcome Prediction in Non-Small Cell Lung Cancer. Radiology (2019) 293 (2):190357. doi: 10.1148/radiol.2019190357

12. Sun R, Limkin EJ, Vakalopoulou M, Laurent D, Stéphane C, Rong HS, et al. A Radiomics Approach to Assess Tumour-Infiltrating CD8 Cells and Response to Anti-PD-1 or Anti-PD-L1 Immunotherapy: An Imaging Biomarker, Retrospective Multicohort Study. Lancet Oncol (2018) 19:S1470204518304133. doi: 10.1016/S1470-2045(18)30413-3

13. Dong D, Tang L, Li ZY, Fang MJ, Gao JB, Shan XH, et al. Development and Validation of an Individualized Nomogram to Identify Occult Peritoneal Metastasis in Patients With Advanced Gastric Cancer. Ann Oncol (2019) 30 (3):431-8. doi: 10.1093/annonc/mdz001 
14. Liu S, He J, Liu S, Guan W, Chen L, Guan Y, et al. Radiomics Analysis Using Contrast-Enhanced CT for Preoperative Prediction of Occult Peritoneal Metastasis in Advanced Gastric Cancer. Eur Radiol (2020) 30:239-46. doi: 10.1007/s00330-019-06368-5

15. Guo HL, Ling H, Zhu YC, Wu K, Feng Y. Comparison Between Multi-Slice Spiral CT and Magnetic Resonance Imaging in the Diagnosis of Peritoneal Metastasis in Primary Ovarian Carcinoma. Onco Targets Ther (2018) 11:1087-94. doi: 10.2147/OTT.S147700

16. Thomassin-Naggara I, Aubert E, Rockall A, Jalaguier-Coudray A, Rouzier R, Darai E, et al. Adnexal Masses: Development and Preliminary Validation of an MR Imaging Scoring System. Radiology (2013) 267(2):432-43. doi: 10.1148/ radiol.13121161

17. Qian LD, Ren JL, Liu AS, Gao Y, Niu GM. MR Imaging of Epithelial Ovarian Cancer: A Combined Model to Predict Histologic Subtypes. Eur Radiol (2020) 18(30):5815-25. doi: 10.1007/s00330-020-06993-5

18. Cohen MS, Dubois RM, Zeineh MM. Rapid and Effective Correction of RF Inhomogeneity for High Field Magnetic Resonance Imaging. Hum Brain Mapp (2015) 10(4):204-11. doi: 10.1002/1097-0193(200008)10:4<204::AIDHBM60>3.0.CO;2-2

19. Griethuysen J, Fedorov A, Parmar C, Hosny A, Aerts HJWL. Computational Radiomics System to Decode the Radiographic Phenotype. Cancer Res (2017) 77(21):e104-7. doi: 10.1158/0008-5472.CAN-17-0339

20. Parmar C, Grossmann P, Rietveld D, Rietbergen MM, Lambin P, Aerts Hugo JWL. Radiomic Machine Learning Classifiers for Prognostic Biomarkers of Head \& Neck Cancer. Front Oncol (2015) 5(4):272. doi: 10.3389/ fonc.2015.00272

21. Parmar C, Grossmann P, Bussink J, Lambin P, Aerts H. Machine Learning Methods for Quantitative Radiomic Biomarkers. Rep (2015) 5:13087. doi: $10.1038 /$ srep 13087

22. Peng H, Long F, Ding C. Feature Selection Based on Mutual Information: Criteria of Max-Dependency, Max-Relevance, and Min-Redundancy. IEEE Trans Pattern Anal Mach Intell (2005) 27:1226-38. doi: 10.1109/ TPAMI.2005.159

23. Huang Y-Q, Liang C-H, He L, Tian J, Liang CS, Chen X, et al. Development and Validation of a Radiomics Nomogram for Preoperative Prediction of Lymph Node Metastasis in Colorectal Cancer. Sci Foundation China (2016) 34 (18):2157-64. doi: 10.1200/JCO.2015.65.9128

24. Grabowska-Derlatka L, Derlatka P, Szeszkowski W, Cieszanowski A, Wang Y-X. Diffusion-Weighted Imaging of Small Peritoneal Implants in "Potentially" Early-Stage Ovarian Cancer. BioMed Res Int (2016) 2016:9254742-9254742. doi: 10.1155/2016/9254742

25. Rubini G, Altini C, Notaristefano A, Merenda N, Rubini D, Ianora AAS, et al. Role of 18F-FDG PET/CT in Diagnosing Peritoneal Carcinomatosis in the Restaging of Patient With Ovarian Cancer as Compared to Contrast Enhanced CT and Tumor Marker Ca-125. Rev Esp Med Nucl Imagen Mol (2014) 33(1):22-7. doi: 10.1016/j.remn.2013.06.008
26. Zhang B, Tian J, Dong D, Dongsheng G, Shuixing Z. Radiomics Features of Multiparametric MRI as Novel Prognostic Factors in Advanced Nasopharyngeal Carcinoma. Clin Cancer Res (2017) 23(15):4259-69. doi: 10.1158/1078-0432.CCR-16-2910

27. Chen, Qin, Nie, Ke, Niu, Tianye, et al. Rectal Cancer: Assessment of Neoadjuvant Chemoradiation Outcome Based on Radiomics of Multiparametric MRI. Clin Cancer Res (2016) 22(21):5256-64. doi: 10.1158/ 1078-0432.CCR-15-2997

28. Thrall Melissa M, DeLoia Julie A, Gallion H, Avril N. Clinical Use of Combined Positron Emission Tomography and Computed Tomography (FDG-PET/CT) in Recurrent Ovarian Cancer. Gynecol Oncol (2007) 105 (1):17-22. doi: 10.1016/j.ygyno.2006.10.060

29. Bilici A, Ustaalioglu B, Seker M, Nesrin C, Bulent T, Taflan S, et al. Clinical Value of FDG PET/CT in the Diagnosis of Suspected Recurrent Ovarian Cancer: Is There an Impact of FDG PET/CT on Patient Management? Eur J Nucl Med Mol Imaging (2010) 37(7):1259-69. doi: 10.1007/s00259-010-1416-2

30. Chen D, Liu Z, Liu W, Fu M, Jiang W, Xu S, et al. Predicting Postoperative Peritoneal Metastasis in Gastric Cancer With Serosal Invasion Using a Collagen Nomogram. Nat Commun (2021) 12(1):179. doi: 10.1038/s41467-020-20429-0

31. Arakawa, Keiichi, Kawai, Kazushige, Ishihara, Soichiro, et al. Prognostic Significance of Peritoneal Metastasis in Stage IV Colorectal Cancer Patients With R0 Resection: A Multicenter, Retrospective Study. Dis Colon Rectum (2017) 60l:1041-9. doi: 10.1097/DCR.0000000000000858

32. Werner ME, Karve S, Sukumar R, Cummings ND, Copp JA, Chen RC, et al. Folate-Targeted Nanoparticle Delivery of Chemo- and Radiotherapeutics for the Treatment of Ovarian Cancer Peritoneal Metastasis. Biomaterials (2011) 32(33):8548-54. doi: 10.1016/j.biomaterials.2011.07.067

Conflict of Interest: Author JR was employed by GE Healthcare.

The remaining authors declare that the research was conducted in the absence of any commercial or financial relationships that could be construed as a potential conflict of interest.

Publisher's Note: All claims expressed in this article are solely those of the authors and do not necessarily represent those of their affiliated organizations, or those of the publisher, the editors and the reviewers. Any product that may be evaluated in this article, or claim that may be made by its manufacturer, is not guaranteed or endorsed by the publisher.

Copyright (c) 2021 Yu, Ren, Jia, Wu, Niu, Liu, Gao, Hao and Xie. This is an open-access article distributed under the terms of the Creative Commons Attribution License (CC BY). The use, distribution or reproduction in other forums is permitted, provided the original author(s) and the copyright owner(s) are credited and that the original publication in this journal is cited, in accordance with accepted academic practice. No use, distribution or reproduction is permitted which does not comply with these terms. 